\title{
The association between body shape silhovette and dietary pattern among Mexican women
}

\author{
Isabelle Romieu ${ }^{1,2, *} \dagger$, María C Escamilla-Núñez ${ }^{2}$, Luisa M Sánchez-Zamorano², \\ Ruy Lopez-Ridaura ${ }^{2}$, Gabriela Torres-Mejía ${ }^{2}$, Elsa M Yunes ${ }^{2}$, Martin Lajous ${ }^{2}$, \\ Juan A Rivera-Dommarco ${ }^{2}$ and Eduardo Lazcano-Ponce ${ }^{2}$ \\ ${ }^{1}$ International Agency for Research on Cancer, Lyon, France: ${ }^{2}$ Instituto Nacional de Salud Pública, \\ Av. Universidad \# 655, Col. Santa María Ahuacatitlán, C. P. 62100, Cuernavaca, Morelos, Mexico
}

Submitted 5 October 2010: Accepted 14 April 2011: First published online 30 August 2011

\begin{abstract}
Objective: To investigate the relationship between dietary patterns and selfperceived body shape silhouette and BMI in a sample of Mexican women.

Design: A cross-sectional analysis of dietary habits from baseline data of a large cohort study (EsMaestra) conducted in 2006-2008.

Setting: The state of Veracruz, Mexico.

Subjects: Mexican teachers ( $n$ 20330) provided information on body shape silhouette at baseline, changes in body shape silhouette and BMI, as well as information on sociodemographic variables and lifestyle.

Results: The median BMI was $26 \cdot 8 \mathrm{~kg} / \mathrm{m}^{2} ; 43 \%$ of women were overweight and $24 \%$ were obese. The carbohydrates, sweet drinks and refined foods pattern was associated with a greater risk of having a large silhouette and a large BMI (BMI $\geq$ $30 \cdot 0 \mathrm{~kg} / \mathrm{m}^{2} v$. BMI $<25 \cdot 0 \mathrm{~kg} / \mathrm{m}^{2} ; \mathrm{OR}_{\mathrm{T} 1-3}=1 \cdot 86,95 \% \mathrm{CI} 1 \cdot 56,2 \cdot 22$ and $1 \cdot 47,95 \% \mathrm{CI}$ $1 \cdot 28,1 \cdot 69$, respectively) with a significant trend when comparing the first and third tertiles of intake. The fruit and vegetable pattern was associated with a lower risk of having a large silhouette and a large BMI $\left(\mathrm{OR}_{\mathrm{T} 1-3}=0 \cdot 68,95 \% \mathrm{CI} 0 \cdot 57,0 \cdot 82\right.$ and $\mathrm{OR}_{\mathrm{T} 1-3}=0 \cdot 77,95 \% \mathrm{CI} 0 \cdot 67,0 \cdot 88$, respectively) with a significant decreasing trend. Similar results were observed when change in silhouette (from 18 years of age to current silhouette) was considered.

Conclusions: High intakes of carbohydrates, sweet drinks and refined foods are related to larger silhouettes. Public health intervention improving access to healthy dietary guidelines, healthy food choice in the work place, promotion of physical activity and regulation of beverages with a high sugar content and of refined foods should be considered.
\end{abstract}

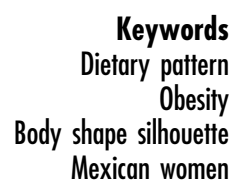

Lifestyle and dietary habits in the Mexican population have changed dramatically in the past 20 years, reflected in an increased prevalence of overweight and obesity in both urban and rural adult populations - from $33 \cdot 4 \%$ in 1988 to $59 \cdot 6 \%$ in 2000 (Mexican National Health and Nutrition Survey ${ }^{(1)}$ ) and to $71.9 \%$ in $2006^{(2)}$. This represents a $41 \%$ and $160 \%$ increase in prevalence of overweight and obesity, respectively, in just a decade ${ }^{(3)}$. Among adults, obesity is more prevalent among women than among men. In 2006, 36.9\% of women were obese $\left(\mathrm{BMI} \geq 30 \cdot 0 \mathrm{~kg} / \mathrm{m}^{2}\right)$, whereas only $23 \cdot 5 \%$ of men belonged to the obese group $^{(3)}$. A total of $35 \cdot 1 \%$ of Mexican adult women are estimated to be at risk for excessive carbohydrate intake and $12 \cdot 6 \%$ are deemed to be at risk

$\dagger$ Dr Romieu's actual position belongs to International Agency for Research on Cancer. for excessive fat intake ${ }^{(4)}$. In women over 60 years of age, overweight and obesity rates are even higher - approximately $40 \%$ are overweight and $35.5 \%$ are obese - and a high prevalence of hypertension and diabetes is observed ${ }^{(5)}$. Moreover, it appears that Mexicans have genetic susceptibility to insulin resistance and to altered carbohydrate and lipid metabolism ${ }^{(6)}$. It is therefore important to determine dietary factors that are most strongly associated with obesity in this population to provide public awareness and prevention strategies.

Assessment of dietary patterns is an approach that analyses intakes of specific foods in the context of the whole $\operatorname{diet}^{(7)}$ and may be of particular interest to public health, providing a basis to make recommendations for eating practices that prevent disease. Several dietary patterns have been previously defined and associated with chronic diseases ${ }^{(8-13)}$; dietary patterns are culturally 
defined, however, and differ between ethnically diverse populations. There exist very few data on dietary patterns associated with obesity in the Mexican population. Recently, Carrera et $a l .{ }^{(14)}$ evaluated data from the National Health and Nutrition Examination Survey (NHANES) on a small subsample of 659 Mexican-American adults without identifying a specific dietary pattern associated with obesity in this population.

Body shape silhouette is a promising tool to examine body size and image. Studies conducted in different countries have shown that the simple use of silhouette could adequately rank individuals according to body size $^{(15-18)}$. In a study conducted in Mexico, a very good correlation $(0.77)$ was observed between BMI and classification of body shape silhouette among adult women ${ }^{(19)}$. This approach has special value in large populations for which anthropometric measurements cannot be obtained.

As part of a large follow-up study of Mexican women to investigate the role of diet and lifestyle in the risk for chronic diseases, the association of self-perceived body shape silhouette and BMI with dietary patterns was evaluated. The dietary pattern most strongly associated with obesity and the usefulness of self-perceived body silhouette as proxy for body size in epidemiological studies were also assessed.

\section{Methods}

\section{Study population}

The source population for the present study consisted of a cohort of female teachers from the public education system who were aged $\geq 35$ years and who were active members of the Federal and State level Ministry of Education's Economic Incentives Program called 'Magisterial Career' (MC). Using databases provided by the MC state programmes, we obtained the full name and place of work for active participants in the MC programme, identifying a total of 27456 female teachers who met the age inclusion criterion ( $\geq 35$ years old) in the state of Veracruz, a representative state of the Mexican population. These women work in 9699 different schools distributed across the entire geographical area, including urban and rural areas.

All 27456 women were invited to participate in a cohort study to evaluate lifestyle and chronic disease incidence. In addition to being part of the MC programme and being $\geq 35$ years of age, inclusion criteria included their consent to participate in the cohort study with planned long-term follow-up. The rationale for inviting MC programme participants was that MC members are highly motivated and trained individuals who are capable of responding to complex questionnaires in the setting of a developing country. Each potential participant received an invitation letter, a consent form, the baseline questionnaire, a promotional brochure and a fibreglass measuring tape in a personalized sealed envelope. Letters were sent through the state-level MC internal delivery network between 2006 and 2008. The questionnaire was self-administered, and women were asked to return the completed questionnaire to the National Institute of Public Heath in a return envelope provided, ensuring confidentiality. The protocol for the present study was approved by the Ethics Committee of the National Institute of Public Health, the Education Ministry, and the Health Ministry. A total of 20258 questionnaires were returned, corresponding to a participation rate of $73.7 \%$. Respondents and nonrespondents had a similar age distribution and were evenly distributed within the localities (with the exception of the city of Veracruz where respondents represented $15 \%$ of the total sample and non-respondents represented $21 \%$ ). Among respondents, 18875 questionnaires (93.2\%) had complete data on current silhouettes and anthropometric measures. The general characteristics of women did not differ between those who did and those who did not provide complete data on silhouette and anthropometric measures.

\section{Baseline questionnaire}

The questionnaire included general information on demographics, socio-economic status (SES; electrical appliance and car ownership, number of bedrooms and number of persons in the household), reproductive history and use of oral contraceptives, menopausal hormone therapy, clinical history, anthropometry, lifestyle (including an FFQ), physical activity (PA), smoking habits and early-life risk factors.

\section{Anthropometry and silbouettes}

Women were provided with a plastic measuring tape and a short set of instructions to measure their height, waist and hip circumference and weight. Waist-to-hip ratio (WHR) was determined from these measurements. BMI was calculated as weight in kilograms divided by the square of height in metres $\left(\mathrm{kg} / \mathrm{m}^{2}\right)$.

In addition, women reported their weight at 18 years and the maximum weight attained at any age during their lifetime. Women were also asked to select a body silhouette from nine options (from very thin to very fat) at six different ages: 2 years after menarche, between 18 and 20 years of age, before their first pregnancy, between 25 and 35 years of age and at their current age. Responses to these pictograms have been validated in different settings and have proven to be reliable ${ }^{(20)}$.

To validate the results reported on the questionnaire, 1000 women participating in the study were randomly selected and measured, weighed according to recommendations by Lohman et al. ${ }^{(21)}$ and classified according to their body silhouette by a health professional during a clinical examination conducted in 2008. The reliability (concordance) of women reporting their silhouette when compared with that observed by a health professional 
was 0.70 ; the correlation between BMI based on weight and height reported on the questionnaire and that measured during the validation study was $0 \cdot 90$.

\section{FFQ}

Dietary intake was assessed using a semi-quantitative FFQ that included 116 food items with a standard portion size using the Mexican version of that developed by Willett ${ }^{(22)}$. This questionnaire has been validated and has been shown to perform well among women residing in Mexico City ${ }^{(23,24)}$. We added twenty-three food items to evaluate new food consumption patterns. The inclusion of these foods was based on the food reported in $24 \mathrm{~h}$ reports conducted as part of the Mexican National Health Survey ${ }^{(1)}$. For each food item, a commonly used unit or portion size was indicated (specified serving size: slice, glass or natural unit such as one apple) and participants were asked how often, on average over the previous year, they had consumed the specified amount of each food, choosing among ten frequencies of consumption: $\geq 6$ times/d, 4-5 times/d, 2-3 times/d, 1 time/d, 5-6 times/week, 2-4 times/week, 1 time/ week, 2-3 times/month, $\leq 1$ time/month or never. Participants with an unreasonably high intake $(>14644 \mathrm{~kJ}$ $(>3500 \mathrm{kcal}) / \mathrm{d})$ or an unreasonably low intake $(<2510 \mathrm{~kJ}$ $(<600 \mathrm{kcal}) / \mathrm{d})$ and those with more than seventy items left blank were excluded from the analysis ( $n 1752$ or $9 \cdot 3 \%$ ).

\section{Physical activity}

Women were asked to report a representative week in terms of the number of hours of mild, moderate and vigorous activity during work time, work at home or during recreational time on weekdays and weekends, as well as the number of hours of sleep. They were also asked to report the number of hours per week of watching television (TV). Specific definitions and examples were provided to the women on these different types of activities. Hours were summed to calculate the number of hours per week of mild, moderate and vigorous activity and to calculate MET-h/week (MET $=$ metabolic equivalents $)$, using the following coefficient to multiply the number of hours in each specific type of activity: mild $(2 \cdot 2)$, moderate $(4 \cdot 7)$ and vigorous $(6 \cdot 0)$.

\section{Dietary pattern}

Forty-eight food groups were defined (see Appendix) but only forty-six groups were included in the analysis because alcohol and atole (a Mexican drink) had an unsuitable distribution and a low consumption in our population. Food items were classified individually when their composition differed substantially from that of other foods or when they represented particular dietary habits, as mentioned by $\mathrm{Hu}^{(25)}$.

\section{Statistical analysis}

Mean and SD were calculated for continuous variables, and frequencies were calculated for categorical variables. When the variable was not normally distributed, we used a transformation (in general, log transformation) to normalize the distribution. Categorical variables were compared using the $\chi^{2}$ test, and continuous variables were calculated using the difference of mean tests. The SES index was calculated using factor analysis including the following variables: electrical appliance, car ownership, number of bedrooms and number of persons in the household. A single factor explained most of the variance. The SES factor was categorized into tertiles to define three levels of SES: low, medium and high.

Dietary pattern was defined using factor analysis as described earlier ${ }^{(25,26)}$. Factor analysis is a type of cluster analysis $^{(27)}$ that determines those features that are most important when classifying a group of items and that generates 'factor scores' representing values of the underlying constructs for use in other analyses ${ }^{(27)}$. In our context, this analysis allowed the grouping of individuals on the basis of food group intakes. Factors were rotated by orthogonal transformation (Varimax) to achieve simpler structures with greater interpretation. The number of factors retained was determined using a diagram for eigenvalues, the scree plot and the percentage of variance explained. Foods that loaded $\geq 0 \cdot 30$ were considered to contribute to the factor, although the value for meaningful loading is arbitrary and we included two foods in the second factor with a load of $0 \cdot 28$ (milk beverages) and $0 \cdot 24$ (milk). The factor score for each pattern was constructed summing observed intakes of the component food items weighted by factor loading. Three major dietary patterns were identified: (i) fruit and vegetables; (ii) meat and dairy; and (iii) carbohydrates, sweet drinks and refined foods. To reduce measurement errors and represent long-term dietary patterns, the cumulative average of pattern scores was calculated and then divided into tertiles.

Current silhouette was categorized into three groups by re-grouping the nine categories provided on the questionnaire: lean women silhouette, 1-3; medium, 4-6; and large, 7-9. For change in silhouette from 18-20 years to current age we also defined three groups: (i) women who did not change silhouette or who decreased silhouette; (ii) women who increased one or two silhouettes; and (iii) women who increased three or more silhouettes. For BMI we used cut-off points as follows: $<25 \cdot 0 \mathrm{~kg} / \mathrm{m}^{2}$, $\geq 25 \cdot 0-<30 \cdot 0 \mathrm{~kg} / \mathrm{m}^{2}$ (overweight) and $\geq 30 \cdot 0 \mathrm{~kg} / \mathrm{m}^{2}$ (obese). For change in BMI from 18-20 years to current age, we categorized the difference into tertiles. Association of dietary pattern with current silhouette and change in silhouette and with current BMI and change in BMI was determined using logistic regression models, adjusting for potential confounders including age, socio-economic level, total energy intake, hours of watching TV and PA. Other potential confounders (marital status, smoking and parity) were not significant - they did not alter the results by $>1 \%$ - and were therefore not included in the final models. All analyses were conducted using the STATA statistical software package version $9 \cdot 2$ (StataCorp., College Station, TX, USA). 


\section{Results}

\section{Population characteristics}

Table 1 presents general and sociodemographic characteristics of the 18875 women included in the analysis.

The mean age was 44 years in a range from 35 to 77 years. Most of the women lived with a partner. Approximately $12 \%$ spoke a native language. Most of the women were non-smokers or former smokers and only 8.5\% reported being current smokers. The majority of women reported having one or two children with an age at first pregnancy between 16 and 39 years. Among teachers with children, $82 \cdot 3 \%$ reported breast-feeding. Women reported watching TV on average $5 \mathrm{~h} /$ week. PA was low; only $13 \cdot 6 \%$ reported some amount of vigorous PA (such as running or playing tennis). The mean moderate activity

Table 1 Sociodemographic characteristics of women from Veracruz; EsMaestras Cohort Study, Mexico

\begin{tabular}{|c|c|c|}
\hline Variable & $n^{*}$ & $\%$ \\
\hline \multicolumn{3}{|l|}{ Age category (years) } \\
\hline $35-40$ & 5648 & $29 \cdot 9$ \\
\hline $41-45$ & 6816 & $36 \cdot 1$ \\
\hline $46-50$ & 3967 & $21 \cdot 0$ \\
\hline $51-55$ & 1623 & $8 \cdot 6$ \\
\hline $56-60$ & 595 & $3 \cdot 2$ \\
\hline$>60$ & 226 & $1 \cdot 2$ \\
\hline \multicolumn{3}{|l|}{ Socio-economic level } \\
\hline Low & 6218 & $34 \cdot 6$ \\
\hline Medium & 5300 & $29 \cdot 8$ \\
\hline High & 6275 & $35 \cdot 3$ \\
\hline \multicolumn{3}{|l|}{ Native language spoken } \\
\hline No & 16489 & $88 \cdot 5$ \\
\hline Yes & 2137 & $11 \cdot 5$ \\
\hline \multicolumn{3}{|l|}{ Smoking } \\
\hline Never & 12324 & $67 \cdot 5$ \\
\hline Current & 1557 & $8 \cdot 5$ \\
\hline Former & 4372 & $23 \cdot 6$ \\
\hline Watching television (h/week)† & $5 \cdot 1$ & $3 \cdot 1$ \\
\hline Physical activity (MET-h/week)†‡ & $24 \cdot 9$ & $13 \cdot 6$ \\
\hline \multicolumn{3}{|l|}{ Parity } \\
\hline No pregnancy & 1889 & $10 \cdot 3$ \\
\hline \multicolumn{3}{|c|}{ Number of live births (natural or Caesarean) } \\
\hline $1-2$ & 9984 & $62 \cdot 9$ \\
\hline $3-4$ & 5457 & $34 \cdot 4$ \\
\hline$\geq 5$ & 436 & $2 \cdot 8$ \\
\hline \multicolumn{3}{|l|}{ Living with partner } \\
\hline Yes & 13110 & $70 \cdot 3$ \\
\hline No & 5544 & $29 \cdot 7$ \\
\hline Age at first pregnancy (years) $\dagger$ & $24 \cdot 8$ & $4 \cdot 8$ \\
\hline$<20$ & 18 & $1 \cdot 2$ \\
\hline $20-29$ & $24 \cdot 1$ & $2 \cdot 6$ \\
\hline$>29$ & 33 & $2 \cdot 9$ \\
\hline Breast-feeding (months)§ & 10 & 6,19 \\
\hline \multicolumn{3}{|l|}{ Menopausal status } \\
\hline Premenopausal & 13447 & $73 \cdot 8$ \\
\hline Postmenopausal & 4705 & $25 \cdot 8$ \\
\hline
\end{tabular}

MET, metabolic equivalents.

*The total number for each variable shows some discrepancies because of different numbers of missing values.

tData are presented as mean and SD.

‡On the basis of the number of hours of mild, moderate and vigorous activities during work time, work at home or during recreational time on weekdays and weekend days in a representative week, as well as the number of hours of sleep.

\$Data are presented as median and 25th and 75th percentiles. (such as playing volleyball, light bicycling, swimming, walking, dancing and home activities) was $6 \cdot 7$ (sD 3.2) h/ week. In all, $74 \%$ of women were premenopausal and $26 \%$ reported natural menopause; $70 \%$ of women reported living with a partner.

Table 2 presents anthropometric measurements. The median BMI was $26 \cdot 8 \mathrm{~kg} / \mathrm{m}^{2}$ (25th percentile $=24 \cdot 2,75$ th percentile $=30 \cdot 0) ; 67 \%$ of women were either overweight (43\%) or obese (24\%). In addition, women who reported living with a partner were heavier than women who reported being single $(69 \%$ v. 31\% with $\mathrm{BMI} \geq$ $\left.30 \cdot 0 \mathrm{~kg} / \mathrm{m}^{2} ; P=0 \cdot 000\right)$.

\section{Silbouette and change in silbouette}

Overall, $34 \cdot 3 \%$ of women classified themselves as currently having a large body silhouette (7-9) and only $10.9 \%$ as having a thin body silhouette (1-3). This contrasts with reports regarding body silhouette close to menarche, at which age $78.8 \%$ of women reported a thin body silhouette and only $1.6 \%$ reported a large body silhouette. A clear shift over time from a thinner to a larger silhouette was observed (Table 3, Fig. 1).

The majority of women increased silhouette from menarche to their current age, gaining up to three silhouettes over time. The change in silhouette from 18-20 years to current age was less, with the highest proportion of change corresponding to an increase of two silhouettes (25.7\%).

\section{Dietary pattern and body silhouette}

Foods most frequently consumed were tortilla and sweet breads, fruity and leafy vegetables (including tomato), rice and pasta, beans, fruit, milk, cereal, cream and butter. The fruit and vegetable pattern was composed mainly of fruit and vegetables, nuts and cereals. The meat and dairy pattern was composed mainly of meat, processed meat, fish, cream and butter, cheese and milk. The carbohydrates, sweet drinks and refined foods pattern was composed of tortilla and sweet bread, biscuits and pastry, rice and pasta, beans, jam and soft drinks. Table 4 displays factors for particular foods consumed in each of the three intake patterns.

Women with the highest (3rd tertile) intakes of carbohydrates, sweet drinks and refined foods were more likely to have a large body silhouette (7-9) when compared with women with the lowest intake (1st tertile; $1-3$; $\mathrm{OR}_{\mathrm{T} 1-3}=1 \cdot 86 ; 95 \% \mathrm{CI} 1 \cdot 56,2 \cdot 22$, test for trend $P=0 \cdot 001$ ). In addition, women with the highest intakes of carbohydrates, sweet drinks and refined foods were more likely to have increased by $\geq 3$ silhouettes from 18-20 years of age to current age $\left(\mathrm{OR}_{\mathrm{T} 1-3}=1.56 ; 95 \%\right.$ CI 1·31, 1·85, test for trend $P=0 \cdot 10)$.

In contrast, women with the highest fruit and vegetable intakes were less likely to have a large silhouette $\left(\mathrm{OR}_{\mathrm{T} 1-3}=\right.$ $0 \cdot 68 ; 95 \%$ CI $0 \cdot 57,0 \cdot 82$ ) compared with women with the lowest intake, and to increase silhouette by $\geq 3$ from $18-20$ years of age to current age $\left(\mathrm{OR}_{\mathrm{T} 1-3}=0 \cdot 76 ; 95 \% \mathrm{CI} 0 \cdot 64\right.$, 0.90). Significant trends were observed for both outcomes. 
Table 2 Baseline anthropometric measurements of women from Veracruz; EsMaestras Cohort Study, Mexico

\begin{tabular}{|c|c|c|c|}
\hline Anthropometrics & Median & 5th percentile & 95th percentile \\
\hline Weight (kg) & $65 \cdot 0$ & $50 \cdot 0$ & $89 \cdot 0$ \\
\hline Height $(\mathrm{cm})$ & $156 \cdot 0$ & $146 \cdot 0$ & $167 \cdot 0$ \\
\hline $\operatorname{BMI}\left(\mathrm{kg} / \mathrm{m}^{2}\right)$ & $26 \cdot 8$ & $21 \cdot 2$ & $35 \cdot 6$ \\
\hline Overweight $\left(25 \cdot 0 \geq \mathrm{BMI}<30 \cdot 0 \mathrm{~kg} / \mathrm{m}^{2}\right)^{*}$ & $27 \cdot 2$ & $25 \cdot 2$ & $29 \cdot 7$ \\
\hline Obesity $\left(\mathrm{BMI} \geq 30.0 \mathrm{~kg} / \mathrm{m}^{2}\right)^{\star}$ & $32 \cdot 8$ & $30 \cdot 1$ & $40 \cdot 4$ \\
\hline Change of BMI: from 18 years to current & $6 \cdot 7$ & $0 \cdot 76$ & $14 \cdot 7$ \\
\hline Change of silhouette: from $18-20$ years to current & $2 \cdot 0$ & 0.0 & $5 \cdot 0$ \\
\hline
\end{tabular}

${ }^{*}$ Median and 5th and 95th percentiles are presented for women within this category of BMI.

Table 3 Self-perception of body image at baseline and at different ages for women from Veracruz; EsMaestras Cohort Study, Mexico

\begin{tabular}{|c|c|c|c|c|}
\hline \multirow[b]{3}{*}{ Lifetime } & \multirow[b]{3}{*}{$n^{\star}$} & \multicolumn{3}{|c|}{ Self-perception of body image (silhouette) } \\
\hline & & $1-3$ & $4-6$ & $7-9$ \\
\hline & & $\%$ & $\%$ & $\%$ \\
\hline Two years after menarche & 18522 & $78 \cdot 8$ & $19 \cdot 7$ & $1 \cdot 6$ \\
\hline Between 18 and 20 years & 18530 & $66 \cdot 7$ & $31 \cdot 1$ & $2 \cdot 2$ \\
\hline Before first pregnancy $\dagger$ & 16260 & $56 \cdot 6$ & $40 \cdot 3$ & $3 \cdot 1$ \\
\hline Between 25 and 35 years & 18489 & $25 \cdot 9$ & $61 \cdot 8$ & $12 \cdot 3$ \\
\hline Current & 18875 & $10 \cdot 9$ & $54 \cdot 8$ & $34 \cdot 3$ \\
\hline
\end{tabular}

*Some missing values.

tOnly women with parity.

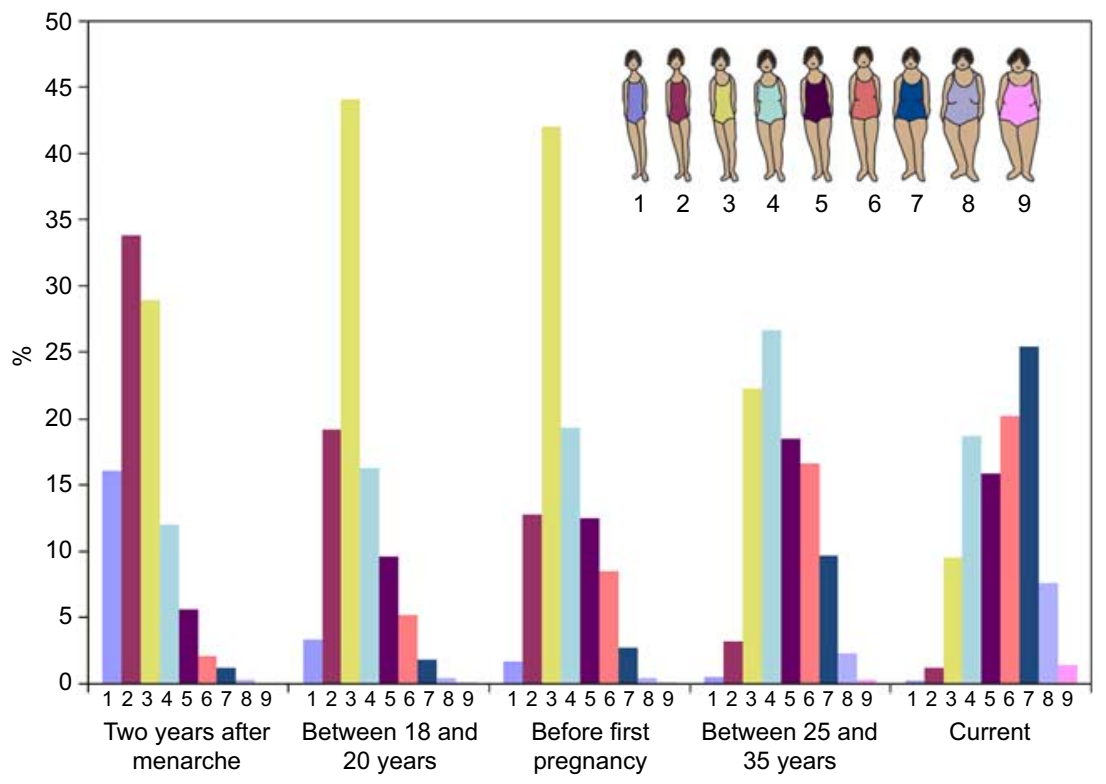

Fig. 1 (colour online) Frequency distribution of women from Veracruz by perceived silhouette at different ages; EsMaestras Cohort Study, Mexico

For the meat and dairy pattern, no significant relation was observed with current silhouette or change in silhouette (Table 5).

\section{Dietary pattern and BMI}

Women with the highest (3rd tertile) intakes of carbohydrates, sweet drinks and refined foods were also more likely to have a large BMI $\left(\geq 30 \cdot 0 \mathrm{~kg} / \mathrm{m}^{2} v .<25 \cdot 0 \mathrm{~kg} / \mathrm{m}^{2}\right)$ when compared with women with the lowest intake (1st tertile), with a significant increasing trend with increasing tertiles of intake $\left(\mathrm{OR}_{\mathrm{T} 1-3}=1 \cdot 47 ; 95 \% \mathrm{CI} 1 \cdot 28\right.$, $1 \cdot 69$, test for trend $P<0 \cdot 00$ ). In addition, women with the highest intakes of carbohydrates, sweet drinks and refined foods were more likely to have a larger increase in BMI, with a significant increasing trend with increasing intake $\left(\mathrm{OR}_{\mathrm{T} 1-3}=1 \cdot 27 ; 95 \% \mathrm{CI} 1 \cdot 11,1 \cdot 44\right.$, test for trend $\left.P=001\right)$.

As observed for silhouette, women with the highest fruit and vegetable intakes were less likely to have a large 
Table 4 Factor-loading matrix for the major factors (dietary patterns) among women ( $n$ 19705) from Veracruz; EsMaestras Cohort Study, Mexico

\begin{tabular}{|c|c|c|c|}
\hline Foods or food groups & Factor 1 & Factor 2 & Factor 3 \\
\hline Fruity vegetables & $0 \cdot 81$ & & \\
\hline Carrots & $0 \cdot 76$ & & \\
\hline Other fruits & 0.73 & & \\
\hline Leafy vegetables (except cabbage) & $0 \cdot 64$ & & \\
\hline Fruits with $\beta$-carotene & $0 \cdot 61$ & & \\
\hline Potatoes & 0.59 & & \\
\hline Legumes & 0.58 & & \\
\hline Elote & 0.58 & & \\
\hline Cabbage & 0.58 & & \\
\hline Fruits with citric acid & 0.58 & & \\
\hline Orange juice & 0.52 & & \\
\hline Breakfast cereals & 0.49 & & \\
\hline Nuts & 0.46 & & \\
\hline Onion & 0.43 & & \\
\hline Waters & $0 \cdot 30$ & & \\
\hline Red meat & & 0.65 & \\
\hline Processed meat & & $0 \cdot 61$ & \\
\hline Fish & & 0.54 & \\
\hline Seafood & & 0.53 & \\
\hline Butter & & 0.51 & \\
\hline Cream & & 0.49 & \\
\hline Cheese & & 0.48 & \\
\hline Offal & & 0.48 & \\
\hline Fast food & & 0.47 & \\
\hline Ice cream & & 0.44 & \\
\hline Yoghurt & & 0.44 & \\
\hline Mexican food & & 0.43 & \\
\hline Semi-skimmed cheese & & 0.43 & \\
\hline Tuna and sardines & & 0.42 & \\
\hline Antojitos mexicanos & & 0.42 & \\
\hline Margarines & & $0 \cdot 39$ & \\
\hline Poultry & & $0 \cdot 36$ & \\
\hline Milk beverages & & $0 \cdot 28$ & \\
\hline Milk & & 0.24 & \\
\hline Cakes, pies, pastries & & & 0.69 \\
\hline Junk food & & & 0.59 \\
\hline Dry cakes, biscuits & & & 0.53 \\
\hline Salty biscuits, aperitif biscuits & & & 0.51 \\
\hline Bread & & & 0.51 \\
\hline Pasta and rice & & & 0.49 \\
\hline Chocolate & & & 0.49 \\
\hline Tortilla & & & $0 \cdot 41$ \\
\hline Gelatin & & & $0 \cdot 39$ \\
\hline Coffee and tea & & & $0 \cdot 37$ \\
\hline Chiles & & & 0.37 \\
\hline Carbonated/soft/isotonic drinks & & & $0 \cdot 37$ \\
\hline
\end{tabular}

Factor 1, fruit and vegetables pattern; Factor 2, meat and dairy pattern Factor 3, carbohydrates, sweet drinks and refined foods pattern.

BMI and to increase BMI between 18 years and current age, with a significant trend with increasing tertiles of intake $\left(\mathrm{OR}_{\mathrm{T} 1-3}=0 \cdot 77 ; 95 \% \mathrm{CI} 0 \cdot 67,0 \cdot 88\right.$ and $\mathrm{OR}_{\mathrm{T} 1-3}=$ $0 \cdot 79 ; 95 \%$ CI $0 \cdot 69,0 \cdot 90$, respectively). No clear effect was observed for the meat and dairy pattern (Table 6).

\section{Discussion}

A dietary pattern characterized by high intakes of carbohydrates, sweet drinks and refined foods was significantly associated with current large body size and obesity. Significant trends were observed with increasing tertiles of intake. In contrast, a dietary pattern characterized by high intakes of fruit, vegetables, grains and nuts appears to protect against large body size and obesity. These patterns also appeared to be related to a change in silhouette over time.

The prevalence of obesity and overweight is increasing in the Mexican population ${ }^{(28)}$ and this trend is made apparent by comparing national nutritional surveys conducted over the past 20 years. Overweight and obesity affect approximately $70 \%$ of the Mexican population aged between 30 and 60 years for both sexes, with a greater percentage of obesity (approximately 32\%) among women (BMI $\geq 30 \cdot 0$ $\mathrm{kg} / \mathrm{m}^{2}$ ). In our study, women from the state of Veracruz were included for strategic reasons: Veracruz had been the first state to be included in our teachers' cohort. In all, $61 \%$ of the teachers were either overweight (39\%) or obese (22\%). The National Nutrition and Health Survey $2006^{(2)}$ is a cross-sectional survey conducted on a representative sample of the Mexican population and included objective anthropometric measurements among other evaluations ( $n 33624$ participants, among whom 9848 women were $\geq 20$ years old). In that study, the median BMI was $26 \cdot 8 \mathrm{~kg} / \mathrm{m}^{2}$, similar to our results ${ }^{(4,28)}$.

High intakes of carbohydrates, sweet drinks and refined foods were strongly related to larger silhouettes and BMI. Although we were able to measure only current diet, our results also suggest that this dietary pattern was related to an increase in silhouette and BMI over time. This pattern was highly weighted by fast-absorbed carbohydrates (simple carbohydrates), in particular sugarsweetened soft drinks, which are major contributors to energy intake and have been associated with high body fat and increased weight gain in other populations ${ }^{(14,29)}$. The expert committee in charge of developing the beverage consumption recommendations for the Mexican population has observed that beverages contribute to one-fifth of all energy consumed by Mexicans and are strongly related to obesity. Among the biggest contributors are beverages high in sugar and low in nutritional value (soft drinks and other beverages with significant amounts of added sugar, such as juices, flavoured water, coffee and tea ${ }^{(30)}$.

In our population, an intake pattern rich in fruits, vegetables and cereals was associated with a thin figure, low BMI and the smallest change in silhouette and gain in BMI over time. Other studies have observed that a 'prudent pattern' with high intakes of fruit and vegetables is negatively associated with higher BMI ${ }^{(31)}$. In prospective settings, Newby et al. reported an association of a diet high in reduced-fat dairy products, whole grains and fruit and low in refined grains, processed and red meat, fast food and soda with smallest gain in BMI and waist circumference ${ }^{(11,32)}$.

Two recent studies have evaluated dietary patterns in Mexican Americans and identified differences among them. In the NHANES 2001-2002, which included 659 Mexican-American adults, four dietary patterns were identified: poultry and alcohol, milk and baked products, traditional Mexican food and meat. None of these patterns 
Table 5 Association of current silhouette and change in silhouette with dietary patterns in women from Veracruz; the EsMaestras Cohort Study, Mexico

\begin{tabular}{|c|c|c|c|c|}
\hline & \multicolumn{3}{|c|}{ Dietary pattern* } & \multirow[b]{2}{*}{$P$ valuet } \\
\hline & Low (tertile 1) & Moderate (tertile 2) & High (tertile 3) & \\
\hline Current silhouette & \multicolumn{3}{|c|}{ Fruit and vegetables pattern } & \\
\hline \multicolumn{5}{|l|}{ Largest $(7-9) v$. thinnest $(1-3)$} \\
\hline $\begin{array}{l}\text { OR } \\
95 \% \mathrm{Cl}\end{array}$ & $1 \cdot 00$ & $\begin{array}{l}0.92 \\
0.80,1.07 \\
\text { Meat and dairy patterr }\end{array}$ & $\begin{array}{c}0.68 \\
0.57,0.82\end{array}$ & 0.000 \\
\hline Largest $(7-9) v$. thinnest $(1-3)$ & \multicolumn{3}{|c|}{ Meat and dairy pattern } & \\
\hline $\begin{array}{l}\mathrm{OR} \\
95 \% \mathrm{Cl}\end{array}$ & $1 \cdot 00$ & $\begin{array}{c}1 \cdot 07 \\
0.93,1 \cdot 24\end{array}$ & $\begin{array}{c}1 \cdot 06 \\
0.90,1 \cdot 24\end{array}$ & $0 \cdot 876$ \\
\hline Largest $(7-9) v$. thinnest $(1-3)$ & \multicolumn{3}{|c|}{ Carbohydrates, sweet drinks and refined foods pattern } & \\
\hline $\begin{array}{l}\text { OR } \\
95 \% \mathrm{Cl}\end{array}$ & $1 \cdot 00$ & $\begin{array}{c}1 \cdot 43 \\
1 \cdot 24,1 \cdot 64\end{array}$ & $\begin{array}{c}1 \cdot 86 \\
1 \cdot 56,2 \cdot 22\end{array}$ & 0.001 \\
\hline Change of silhouette & \multicolumn{3}{|c|}{ Fruit and vegetables pattern } & \\
\hline $\begin{array}{l}\text { Change of } \geq 3 \text { silhouettes } v \text {. no change or decreased silhouette } \\
\text { OR } \\
95 \% \mathrm{Cl}\end{array}$ & $1 \cdot 00$ & $\begin{array}{c}0.99 \\
0 \cdot 86,1 \cdot 14 \\
\text { Meat and dairy patterr }\end{array}$ & $\begin{array}{c}0.76 \\
0.64,0.90\end{array}$ & 0.000 \\
\hline $\begin{array}{l}\text { Change of } \geq 3 \text { silhouettes } v \text {. no change or decreased silhouette } \\
\text { OR } \\
95 \% \mathrm{Cl}\end{array}$ & $1 \cdot 00$ & $\begin{array}{c}1 \cdot 15 \\
1 \cdot 00,1 \cdot 32\end{array}$ & $\begin{array}{l}1 \cdot 21 \\
1 \cdot 04,1 \cdot 42\end{array}$ & $0 \cdot 443$ \\
\hline $\begin{array}{l}\text { Change of } \geq 3 \text { silhouettes } v \text {. no change or decreased silhouette } \\
\text { OR } \\
95 \% \mathrm{Cl}\end{array}$ & Carbohydrates & sweet drinks and refin & $\begin{array}{c}1 \cdot 56 \\
1 \cdot 31,1 \cdot 85\end{array}$ & $0 \cdot 100$ \\
\hline
\end{tabular}

All models were adjusted for age, socio-economic level, total energy intake, hours spent watching television and physical activiy in MET-h/week (MET = metabolic equivalents).

${ }^{*}$ Data are presented as multivariate $\mathrm{OR}$ and $95 \% \mathrm{Cl}$

tTest for trend.

Table 6 Association of current BMl and change in BMI with dietary pattern in women from Veracruz; the EsMaestras Cohort Study, Mexico

\begin{tabular}{|c|c|c|c|c|}
\hline & \multicolumn{4}{|c|}{ Dietary pattern* } \\
\hline & Low (tertile 1) & Moderate (tertile 2) & High (tertile 3) & $P$ valuet \\
\hline Current BMI & \multicolumn{4}{|c|}{ Fruit and vegetables pattern } \\
\hline $\begin{array}{l}\geq 30 \cdot 0 \mathrm{~kg} / \mathrm{m}^{2} \mathrm{v} .<25 \cdot 0 \mathrm{~kg} / \mathrm{m}^{2} \\
\text { OR } \\
95 \% \mathrm{Cl}\end{array}$ & $1 \cdot 00$ & $\begin{array}{l}0.92 \\
0.83,1.03 \\
\text { Meat and dai }\end{array}$ & $\begin{array}{l}0 \cdot 77 \\
0 \cdot 67,0 \cdot 88 \\
\text { tern }\end{array}$ & 0.003 \\
\hline $\begin{array}{l}\geq 30 \cdot 0 \mathrm{~kg} / \mathrm{m}^{2} \mathrm{v} \cdot<25 \cdot 0 \mathrm{~kg} / \mathrm{m}^{2} \\
\quad \text { OR } \\
\quad 95 \% \mathrm{Cl}\end{array}$ & $\mathrm{Ca}$ & $\begin{array}{c}1 \cdot 08 \\
0.96,1 \cdot 20 \\
\text { drates, sweet drinks }\end{array}$ & $\begin{array}{c}1 \cdot 05 \\
0.93,1 \cdot 19 \\
\text { efined foods patt }\end{array}$ & $0 \cdot 702$ \\
\hline $\begin{array}{l}\geq 30 \cdot 0 \mathrm{~kg} / \mathrm{m}^{2} v \cdot<25 \cdot 0 \mathrm{~kg} / \mathrm{m}^{2} \\
\text { OR } \\
95 \% \mathrm{Cl}\end{array}$ & $1 \cdot 00$ & $\begin{array}{l}1 \cdot 15 \\
1 \cdot 03,1 \cdot 29\end{array}$ & $\begin{array}{c}1 \cdot 47 \\
1 \cdot 28,1 \cdot 69\end{array}$ & 0.000 \\
\hline 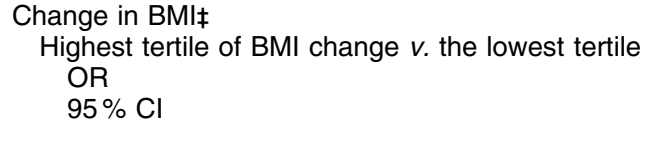 & $1 \cdot 00$ & $\begin{array}{l}0.95 \\
0.85,1 \cdot 05 \\
\text { Meat and dai }\end{array}$ & $\begin{array}{l}0.79 \\
0.69,0.90 \\
\text { tern }\end{array}$ & 0.001 \\
\hline $\begin{array}{l}\text { Highest tertile of BMI change } v \text {. the lowest tertile } \\
\text { OR } \\
95 \% \mathrm{Cl}\end{array}$ & $1 \cdot 00$ & $\begin{array}{c}1 \cdot 06 \\
0.96,1 \cdot 17 \\
\text { drates, sweet drinks }\end{array}$ & $\begin{array}{c}1 \cdot 15 \\
1 \cdot 02,1 \cdot 29 \\
\text { efined foods patt }\end{array}$ & $0 \cdot 127$ \\
\hline $\begin{array}{l}\text { Highest tertile of BMI change } v \text {. the lowest tertile } \\
\text { OR } \\
95 \% \mathrm{Cl}\end{array}$ & $1 \cdot 00$ & $\begin{array}{c}1 \cdot 06 \\
0 \cdot 95,1 \cdot 17\end{array}$ & $\begin{array}{c}1 \cdot 27 \\
1 \cdot 11,1 \cdot 44\end{array}$ & 0.001 \\
\hline
\end{tabular}

All models were adjusted for age, socio-economic level, total energy intake, hours spent watching television and physical activity in MET-h/week (MET = metabolic equivalents).

${ }^{*}$ Data are presented as multivariate OR and $95 \% \mathrm{Cl}$.

tTest for trend.

‡Tertiles of BMl: $-4 \cdot 9-4 \cdot 8 \mathrm{~kg} / \mathrm{m}^{2} ;>4 \cdot 8-8 \cdot 3 \mathrm{~kg} / \mathrm{m}^{2}$ and $>8 \cdot 3-30 \cdot 5 \mathrm{~kg} / \mathrm{m}^{2}$. 
was specifically related to obesity. The traditional Mexican pattern had a high percentage of energy from tortillas, tacos, flavoured and sweetened drinks and legumes. Surprisingly, no 'healthy' pattern group was identified in this population. The small sample size and assessment of dietary intake based on $24 \mathrm{~h}$ recall may explain the lack of significant findings. That analysis with NHANES data as well as ours observed low levels of leisure-time PA. In another study conducted among women living in southwestern USA, including 871 Hispanic women and 1599 nonHispanic women, five dietary patterns were determined: Western, native Hispanic, prudent, Mediterranean and dieter. The native Hispanic pattern was heavily loaded with Mexican cheese, soups, meat, legumes, tomato sauce and sugar-sweetened drinks. Although not significant, the highest tertile of a native Mexican diet was related to a 64\% increased risk of being obese $(95 \% \mathrm{CI} 0 \cdot 83,3 \cdot 18)$ and the highest tertile of a prudent diet was related to a decreased risk of being obese $(\mathrm{OR}=0.54 ; 95 \%$ CI $0.29,1 \cdot 03)$. Although these results are concordant with ours, the small sample size of Mexican women might have limited the ability of the study to detect significant associations ${ }^{(7)}$. A recently published study of data from the National Health Survey in Mexico has also observed in a sample of men and women that overweight and obesity are significantly related to a dietary pattern rich in refined foods and sweets ${ }^{(33)}$.

Several issues need to be discussed. A limitation for interpretation of our results is the cross-sectional nature of our data. Current body silhouette and BMI were reported at the same time as dietary intake; hence, we cannot conclude causality. Heavy women might have gone through changes in dietary pattern to lose weight and may therefore report a diet different from their usual one. However, had this occurred, we would have found a positive association between obesity and the fruit and vegetable pattern, which is not the case. In addition, the consistency of the results using body silhouette, which women appear to underestimate, and BMI strengthens the validity of our results. Our results are consistent with the fact that intake of a diet high in carbohydrates, sweet drinks and refined foods is associated with obesity, whereas a diet rich in fruit and vegetables is protective against obesity. Another limitation is that because dietary intake was reported at baseline, misclassification might have occurred, in particular when considering diet over a long period of time, as in our analysis of change in silhouette. Although previous studies have shown a reasonable tracking of diet over time ${ }^{(34)}$, we do not have data to confirm this in our population. However, our results suggest that dietary pattern may explain part of the change of body silhouette over time, given that random misclassification in dietary pattern would tend to underestimate the association.

The overall consistency of our observations with both body shape silhouette and BMI suggests that in our population women who follow the carbohydrates, sweet drinks and refined foods pattern have a risk factor for large body size and for an increase in body size over time. Body silhouette appears to classify individuals by body size well and hence could be used in epidemiological studies to study obesity and its determinants.

The present study included a large sample of Mexican women, using a validated FFQ to evaluate common diet, anthropometric measurements and self-reported body silhouette. Self-reported body silhouette had a good agreement with observed body silhouette in other studies $^{(15-18)}$. In addition, the validation study showed good agreement between reported and measured BMI and perceived and observed body silhouettes. Must et al. ${ }^{(35)}$ have shown good concordance between recalled and observed body silhouette. Although some misclassifications cannot be avoided, they would most likely be random because women were not influenced by their dietary pattern when reporting body silhouette at different ages. Among Mexican women, knowledge of dietary factors related to obesity is very limited. The association that we observed would therefore be underestimated.

\section{Conclusion}

The significant associations observed between a dietary pattern high in carbohydrates, sweet drinks and refined foods and larger body figures and BMI plus an increase in body figure and BMI over time, as well as the protective effect of a dietary pattern high in fruit and vegetables, strongly support the fact that a diet with a high glycaemic load (corresponding to the carbohydrate content of one serving multiplied by the glycaemic index value of that food) is a risk factor for obesity and weight gain in Mexican women. Such foods, in particular sugarsweetened drinks, pastry and jam, should be restricted. This is particularly important because the intake of carbohydrates is high for the Mexican population (a mean daily carbohydrate intake of $357 \mathrm{~g} / \mathrm{d}$ ), accounting for $64 \%$ of total energy intake ${ }^{(1)}$, whereas the intake of fruit and vegetables is low $(97 \%$ of Mexican women consume $<400 \mathrm{~g} / \mathrm{d}$ ). In addition, Mexicans appear to have a genetic susceptibility to insulin resistance and altered carbohydrate and lipid metabolism ${ }^{(6)}$, with a potential for severe health consequences. As part of a weight-control programme, a decreased intake of quickly absorbed carbohydrates, sweet drinks and refined foods coupled with an increased intake of fruit and vegetables should be promoted along with PA. Several actions could be further developed: improving knowledge on food-based dietary guidelines for our population; providing healthy food choices within canteens and promoting PA such as walking to work and gym activity during lunch break; emphasizing on the responsibility of role modelling of teachers towards students; and supporting the regulation of refined foods, high sugar-sweetened foods and sweet drinks. 


\section{Acknowledgements}

The present study was supported by the American Institute for Cancer Research (Grant no. 05B-047) and by Mexican Sciences and Technology Council (CONACYT; Grant no. 2005-02-14429). The authors have no conflict of interest to declare. I.R. developed the protocol, obtained funding for the project and directed the data analysis and the writing of the manuscript; M.C.E.-N. participated in the protocol and in data collection; L.M.S.-Z. participated in data analysis; R.L. participated in the protocol, in coordination of data collection and in writing of the manuscript; G.T.-M. participated in the protocol and in interpretation of data; E.M.Y. participated in data collection and in standardization of the questionnaires; M.L. participated in the protocol and in interpretation of data; J.A.R.-D and E.L.-P. participated in the interpretation of data and in writing of the manuscript. All authors approved the final report. We are grateful to all participant teachers, who provided invaluable information for the study, for their time and commitment. We would like to thank the Federal Coordinación Nacional de Carrera Magisterial (Coordination Office of the Magisterial Career Program), with special thanks to Victor Sastré, Director of Regulation, Dolores Cruz and María del Carmen Placencia, coordinators from Veracruz and Jalisco States, as well as all their staff for their continued support. We thank also all educational and health authorities who have actively collaborated in the project.

\section{References}

1. Rivera-Dommarco JA, Shamah-Levy T, Villalpando-Hernández S et al. (2001) Encuesta Nacional de Nutrición 1999. Estado nutricio de niños y mujeres en México. Cuernavaca, Morelos: Instituto Nacional de Salud Públic.

2. Olaiz G, Rivera JJ, Shamah T et al. (2006) Encuesta Nacional de Salud y Nutrición 2006. Cuernavaca, Morelos: Instituto Nacional de Salud Publica.

3. Barquera S, Campos-Nonato I, Hernández-Barrera L et al. (2009) Obesity and central adiposity in Mexican adults: results from the Mexican National Health and Nutrition Survey 2006. Salud Publica Mex 51, Suppl. 4, S595-S603.

4. Barquera S, Hernández-Barrera L, Campos-Nonato I et al. (2009) Energy and nutrient consumption in adults: analysis of the Mexican National Health and Nutrition Survey 2006. Salud Publica Mex 51, Suppl. 4, S562-S573.

5. Shamah-Levy T, Cuevas-Nasu L, Mundo-Rosas V et al. (2008) Estado de salud y nutrición de los adultos mayores en México: resultados de una encuesta probabilística nacional. Salud Publica Mex 50, 383-389.

6. Mitchell BD, Blangero J, Comuzzie AG et al. (1998) A paired sibling analysis of the beta-3 adrenergic receptor and obesity in Mexican Americans. J Clin Invest 101, 584-587.

7. Murtaugh MA, Herrick JS, Sweeney C et al. (2007) Diet composition and risk of overweight and obesity in women living in the southwestern United States. J Am Diet Assoc 107, 1311-1321.

8. Fung TT, Hu FB, Holmes MD et al. (2005) Dietary patterns and the risk of postmenopausal breast cancer. Int J Cancer 116, 116-121.
9. Fung TT, Stampfer MJ, Manson JE et al. (2004) Prospective study of major dietary patterns and stroke risk in women. Stroke 35, 2014-2019.

10. Lopez-Garcia E, Schulze MB, Fung TT et al. (2004) Major dietary patterns are related to plasma concentrations of markers of inflammation and endothelial dysfunction. $A m J$ Clin Nutr 80, 1029-1035.

11. Newby PK, Muller D, Hallfrisch J et al. (2003) Dietary patterns and changes in body mass index and waist circumference in adults. Am J Clin Nutr 77, 1417-1425.

12. Sánchez-Villegas A, Bes-Rastrollo M, Martínez-González MA et al. (2006) Adherence to a Mediterranean dietary pattern and weight gain in a follow-up study: the SUN cohort. Int J Obes (Lond) 30, 350-358.

13. Slattery ML, Boucher KM, Caan BJ et al. (1998) Eating patterns and risk of colon cancer. Am J Epidemiol 148, 4-16.

14. Carrera PM, Gao X \& Tucker KL (2007) A study of dietary patterns in the Mexican-American population and their association with obesity. J Am Diet Assoc 107, $1735-1742$.

15. Muñoz-Cachón MJ, Salces I, Arroyo M et al. (2009) Overweight and obesity: prediction by silhouettes in young adults. Obesity (Silver Spring) 17, 545-549.

16. de Lauzon-Guillain B, Balkau B, Charles MA et al. (2010) Birth weight, body silhouette over the life course, and incident diabetes in 91,453 middle-aged women from the French Etude Epidemiologique de Femmes de la Mutuelle Generale de l'Education Nationale (E3N) Cohort. Diabetes Care 33, 298-303.

17. Nagasaka K, Tamakoshi K, Matsushita K et al. (2008) Development and validity of the Japanese version of body shape silhouette: relationship between self-rating silhouette and measured body mass index. Nagoya J Med Sci $\mathbf{7 0}$, 89-96.

18. Tehard B, van Liere MJ, Com Nougué C et al. (2002) Anthropometric measurements and body silhouette of women: validity and perception. J Am Diet Assoc 102, 1779-1784.

19. Kaufer-Horwitz M, Martínez J, Goti-Rodríguez LM et al. (2006) Association between measured BMI and self-perceived body size in Mexican adults. Ann Hum Biol 33, 536-545.

20. Tehard B, van Liere MJ, Com Nougue C et al. (2002) Anthropometric measurements and body silhouette of women: validity and perception. J Am Diet Assoc 102, $1779-1784$.

21. Lohman TG, Roche AF \& Martorell R (1998) Anthropometric Standardization Reference Manual. Champaign, IL: Human Kinetics Publishers, Inc.

22. Willett WC (1998) Nutritional Epidemiology, 2nd ed. New York: Oxford University Press.

23. Hernández-Avila M, Romieu I, Parra S et al. (1998) Validity and reproducibility of a food frequency questionnaire to assess dietary intake of women living in Mexico City. Salud Publica Mex 40, 133-140.

24. Romieu I, Parra S, Hernández JF et al. (1999) Questionnaire assessment of antioxidants and retinol intakes in Mexican women. Arch Med Res 30, 224-239.

25. Hu FB (2002) Dietary pattern analysis: a new direction in nutritional epidemiology. Curr Opin Lipidol 13, 3-9.

26. Varraso R, Fung TT, Barr RG et al. (2007) Prospective study of dietary patterns and chronic obstructive pulmonary disease among US women. Am J Clin Nutr 86, 488-495.

27. Dallas E (1998) Applied Multivariate Methods for Data Analysts. Belmont, CA: Duxbury Press.

28. Shamah-Levy T, Villalpando-Hernández S \& Rivera-Dommarco JA (2007) Resultados de Nutrición de la ENSANUT 2006. Cuernavaca, Morelos: Instituto Nacional de Salud Publica.

29. Novotny R, Daida YG, Acharya S et al. (2004) Dairy intake is associated with lower body fat and soda intake with greater weight in adolescent girls. J Nutr 134, 1905-1909. 
30. Rivera JA, Barquera S, González-Cossío T et al. (2004) Nutrition transition in Mexico and in other Latin American countries. Nutr Rev 62, 7 Pt 2, S149-S157.

31. Togo P, Osler M, Sørensen TI et al. (2001) Food intake patterns and body mass index in observational studies. Int J Obes Relat Metab Disord 25, 1741-1751.

32. Newby PK, Muller D, Hallfrisch J et al. (2004) Food patterns measured by factor analysis and anthropometric changes in adults. Am J Clin Nutr 80, 504-513.
33. Flores M, Macias N, Rivera M et al. (2010) Dietary patterns in Mexican adults are associated with risk of being overweight or obese. J Nutr 140, 1869-1873.

34. Willett WC (1998) Recall of remote diet. In Nutritional Epidemiology, pp. 148-156 [WC Willett, editor]. New York: Oxford University Press.

35. Must A, Willett WC \& Dietz WH (1993) Remote recall of childhood height, weight, and body build by elderly subjects. Am J Epidemiol 138, 56-64.

\section{Appendix}

\begin{tabular}{|c|c|}
\hline Food or food groups & Food items \\
\hline Fruity vegetables & $\begin{array}{l}\text { Courgette, chayote, cucumbers, beet, courgette flower, green beans, tomato sauce, raw tomato, } \\
\text { tomato, avocado, nopal }\end{array}$ \\
\hline Leafy vegetables (except cabbage) & Spinach, lettuce, broccoli \\
\hline Other fruits & $\begin{array}{l}\text { Banana, apple, pear, plums, strawberries, pineapple, raisins, grapes, watermelon, mango, } \\
\text { mamey, tuna, sapote, papaya, guava, jicama }\end{array}$ \\
\hline Carrots & Carrots \\
\hline Fruits with $\beta$-carotene & Peach, melon, mango \\
\hline Potatoes & Potatoes \\
\hline Legumes & Lentils, legumes \\
\hline Elote & Elote \\
\hline Cabbage & Cabbage \\
\hline Fruits with citric acid & Oranges, grapefruit \\
\hline Orange juice & Orange juice \\
\hline Breakfast cereals & Breakfast cereal, high-fibre cereal, oatmeal \\
\hline Nuts & Nuts \\
\hline Onion & Onion \\
\hline Pasta and rice & Pasta and rice \\
\hline Gelatin & Gelatin or pudding \\
\hline Fish & Skipjack, snapper, dried fish \\
\hline Bread & White bread, sliced bread, whole-wheat bread, flour tortilla \\
\hline Water & Flavoured water, natural water \\
\hline Red meat & Sausage, beef, pork, beef jerky, chicharron \\
\hline Processed meat & Bacon, sausage, ham, pork, turkey, ham, other sausage \\
\hline Seafood & Shrimp, octopus \\
\hline Butter & Butter, low-fat butter \\
\hline Cream & Cream \\
\hline Cheese & Manchego cheese, fresh cheese, non-specified cheese \\
\hline Fast food & Hamburger, hot dog, pizza \\
\hline Offal & Liver \\
\hline Ice cream & Ice cream \\
\hline Yoghurt & Yoghurt and danonino \\
\hline Mexican food & Carnitas, barbacoa, birria, pancita, pozole \\
\hline Semi-skimmed cheese & Cream cheese, Oaxaca cheese \\
\hline Tuna and sardines & Canned tuna and sardines \\
\hline Antojitos mexicanos & Tacos, torta, sope, tamal \\
\hline Margarine & Normal margarine, low-fat margarine \\
\hline Poultry & Chicken \\
\hline Milk beverages & Yakult \\
\hline Milk & Whole milk, skimmed milk, semi-skimmed milk, soya milk \\
\hline Cakes, pies, pastries & Cake, sweet bread, cream cake, jam \\
\hline Junk food & Chips, peanuts \\
\hline Dry cakes, biscuits & Dry biscuits \\
\hline Salty biscuits, aperitif biscuits & Salted biscuits \\
\hline Chocolate & Candy bars, chocolate bars \\
\hline Tortilla & Corn tortilla \\
\hline Coffee and tea & Coffee and tea \\
\hline Chilles & Salsa, canned chillies, dried chillies \\
\hline Carbonated/soft/isotonic & Soft drinks, sweetened soft drinks, light soft drinks \\
\hline Atole & Atole \\
\hline Alcohol beverages & Beer, whisky, rum, brandy, wine, tequila, mezcal, aguardiente, pulque \\
\hline
\end{tabular}

